\title{
Hepatocyte nuclear factor $1 b$ is a novel negative regulator of white adipocyte differentiation
}

\author{
Xin Wang ${ }^{\star 1,2}$, Hao Wu ${ }^{1,2}$, Weihua Yu ${ }^{1}$, Jiangzheng Liu' ${ }^{1}$, Jie Peng ${ }^{1}$, Nai Liao ${ }^{1}$, Jieling Zhang ${ }^{1}$, Xiaodi Zhang ${ }^{1}$ and Chunxu Hai ${ }^{1}$
}

Hepatocyte nuclear factor $1 \mathrm{~b}$ (HNF1b) is a transcription factor belonging to the HNF family. We aimed to investigate the role of HNF1b in white adipocyte differentiation. The expression of HNF1b was reduced in white adipose tissue (WAT) of both diet-induced and genetic obese mice and decreased during the process of 3T3-L1 adipocyte differentiation. Downregulation of HNF1b enhanced 3T3-L1 adipocyte differentiation and upregulation of HNF1b inhibited this process. Upregulation of HNF1b inhibited peroxisome proliferator-activated receptor $\gamma$ (PPAR $\gamma$ ) and its target gene expression, while downregulation of HNF1b increased those genes expression. Overexpression of PPAR $\gamma$ suppressed HNF1b upregulation-induced inhibition of adipocyte differentiation. HNF1b can directly bind with the promoter of PPAR $\gamma$ in 3T3-L1 cells, which was decreased after adipogenic differentiation. HNF1b promoted apoptotic and autophagic cell death in early differentiated adipocytes through regulation of cell cycle progress and cell death-related factors, and thus inhibited the process of mitotic clonal expansion (MCE). HNF1b acted as an antioxidant regulator through regulating various antioxidant enzymes via binding with antioxidant response element. Oxidant treatment suppressed HNF1b upregulation-induced inhibition of adipocyte differentiation. Overall, our results suggest that HNF1b is a novel negative regulator of adipocyte differentiation through regulation of PPAR $\gamma$ signaling, MCE and redox state.

Cell Death and Differentiation (2017) 24, 1588-1597; do:10.1038/cdd.2017.85; published online 16 June 2017

Hepatocyte nuclear factor $1 \mathrm{~b}$, also known as vHNF1, HNF1 $\beta$, TCF2 and LF-B3, is a transcription factor belonging to the HNF family, which are highly conserved across species from yeast to human. ${ }^{1}$ Although they were first identified in the liver, HNF1 proteins distribution are not restricted only to liver, ${ }^{2}$ and HNF1, including HNF1a and HNF1b, is also found to be expressed in the kidney, pancreas, gonads, gut, lung and thymus. HNF1b contains a POU-specific (Pit-1, OCT1/2, UNC-86; POUS) and an atypical POU homeodomain $(\mathrm{POUH})$ that mediate sequence-specific DNA binding and recognize the consensus sequence $5^{\prime}$-GTTAATNATTAAC- $3^{\prime} .{ }^{3}$ HNF1b has a role in the embryonic development of liver, kidney, intestine and pancreatic islets through tissue-specific regulation of gene expression. ${ }^{4}$ HNF1b gene mutations causes diabetes, called maturity-onset diabetes of the young (MODY) 5 , which is a form of diabetes characterized by an early age of onset, usually at a mean age of $17-25.8$ years (30-66\%), genital malformations (12.0-62.5\%), and an autosomal dominant mode of inheritance ${ }^{5-9}$ Findings in recent years have shown that variants of HNF1b are associated with type 2 diabetes. ${ }^{10-13}$ Moreover, Stancakova et al. ${ }^{14}$ investigated the effects of 34 genetic risk variants for hyperglycemia/type 2 diabetes on lipoprotein subclasses and particle composition in a large population-based cohort. They found that HNF1b was significantly associated with lipoprotein traits. ${ }^{14}$ However, there is no evidence supporting the role of HNF1b in the regulation of lipid metabolism.

Obesity is a common form of abnormal lipid metabolism, which is closely associated with multiple pathological conditions and diseases, including hypertension, type 2 diabetes, dyslipidemia, kidney disease, heart disease, cancer, obstructive sleep apnea and osteoarthritis. ${ }^{15,16}$ Obesity is usually resulted from enlargement of adipose tissue and excessive accumulation of triglycerides (TG). The adipose tissue, mainly composed of adipocytes, exists in two types in mammals: white adipose tissue (WAT) and brown adipose tissue (BAT). It is a common conception that the main function of WAT is uptake of lipid and synthesis and storage of TG. ${ }^{17}$ Recent evidence proves that WAT is a key endocrine organ that physiologically secretes adipokines and pathophysiologically releases pro-inflammatory cytokines. ${ }^{18}$ It is not surprising that WAT lies at the heart of a complex network in the regulation of a variety of physiological and biochemical processes. ${ }^{19}$

Fat mass is generally controlled by preadipocyte proliferation, adipocyte differentiation and lipolysis. Adipocyte differentiation, also called adipogenesis, has a key role in adipose tissue development. Adipocyte differentiation is a complex process, which is regulated by a battery of molecular pathways in response to adipogenic stimuli. In the current study, we aimed to investigate the role of HNF1b in the regulation of adipocyte differentiation and to explore the possible mechanisms.

\section{Results}

HNF1b expression is reduced in WAT of obese animals and differentiated white adipocytes. We first evaluated the expression pattern of HNF1b in different tissues in animals.

\footnotetext{
${ }^{1}$ Department of Toxicology, Shaanxi Key Lab of Free Radical Biology and Medicine, The Ministry of Education Key Lab of Hazard Assessment and Control in Special Operational Environment, School of Public Health, Fourth Military Medical University, Xi'an 710032, China

*Corresponding author: X Wang, Department of Toxicology, School of Public Health, Fourth Military Medical University, Changle West Road 169, Xi'an 710032, China. Tel: +86 029 84774882; Fax: +86 029 84774882; E-mail: xinwang@fmmu.edu.cn

${ }^{2}$ These authors contributed equally to this work.

Received 17.12.16; revised 01.4.17; accepted 27.4.17; Edited by RA Knight; published online 16.6.17
} 
a
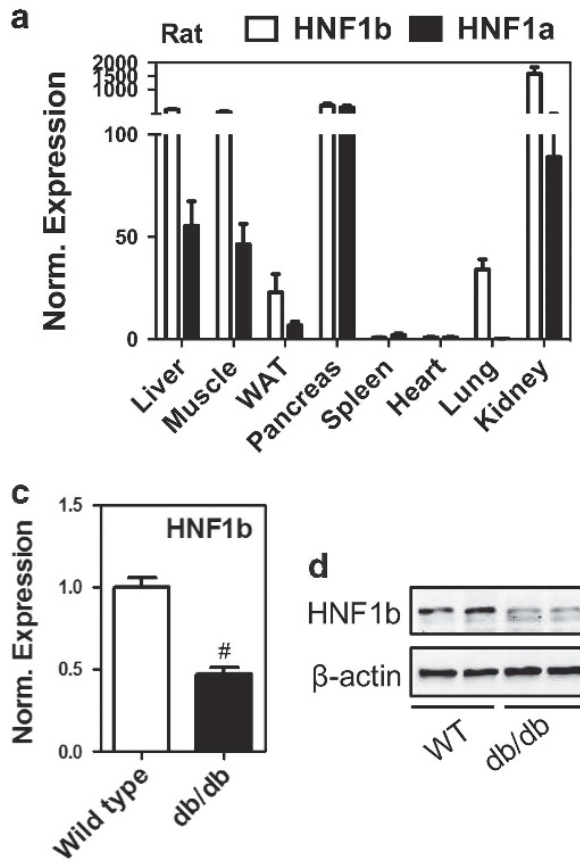

g

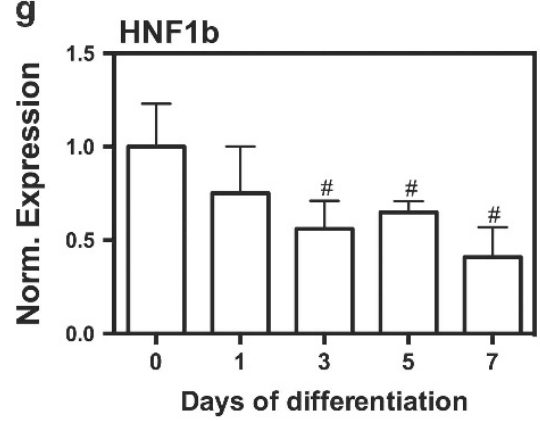

b
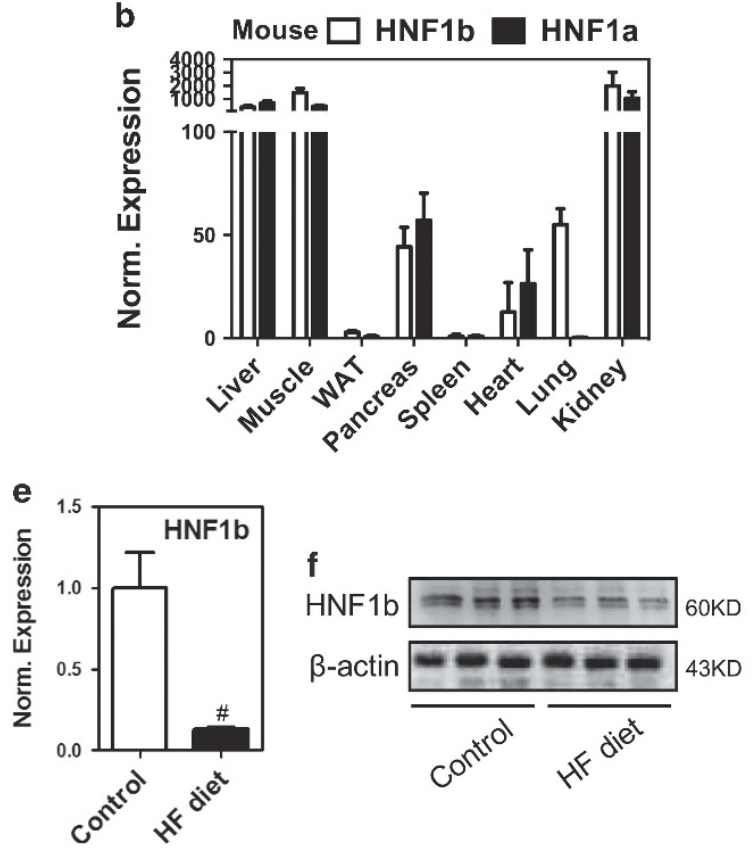

h

HNF1b

$\beta$-actin

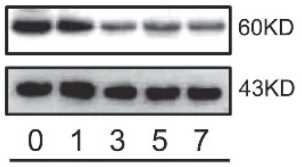

Days of differentiation
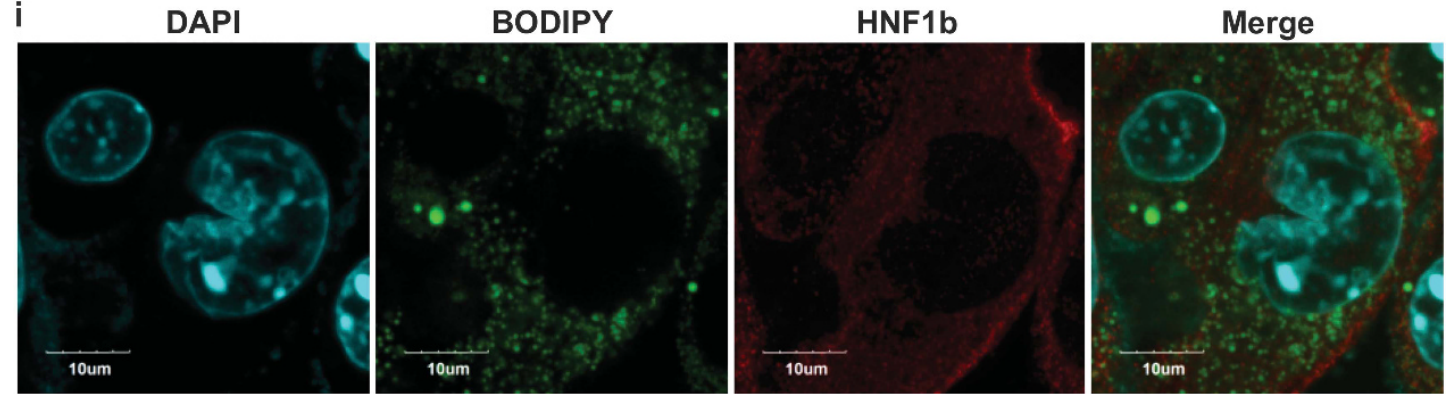

Figure 1 Expression pattern of HNF1b in adipose tissue of animals and during adipocyte differentiation. (a and $\mathbf{b})$ mRNA expression of HNF1b in several tissues in rats and mice were determined. (c and $\mathbf{d}$ ) $\mathrm{mRNA}$ and protein expression of HNF1b in WAT in wild-type and db/db mice were detected. (e and $\mathbf{f}$ ) mRNA and protein expression of HNF1b in WAT in HF diet-fed mice were detected. ( $\mathbf{g}$ and $\mathbf{h}$ ) mRNA and protein expression of HNF1b during 3T3-L1 adipocyte differentiation. (i) Immunostaining of HNF1b and BODIPY staining in differentiated 3T3-L1 adipocytes. ${ }^{\#} P<0.05$, compared with control

We found that HNF1b and its relative family member HNF1a is highly expressed in kidney, liver, muscle and pancreas in both rats and mice (Figures 1a and $b$ ). WAT and spleen have low level of HNF1b and HNF1a expression (Figures 1a and b). Moreover, we detected the expression of HNF1b in WAT of genetic and diet-induced obese mice. The results showed that mRNA and protein expression of HNF1b were significantly decreased in WAT of $\mathrm{db} / \mathrm{db}$ mice and HF diet-induced obese mice, compared with wild-type littermates or control mice (Figures 1c-f), indicating a negative role of HNF1b in the formation of adipose tissue. The expression pattern of HNF1b during the adipocyte differentiation in 3T3-L1 cells was examined. The results showed that mRNA and protein expression of HNF1b were significantly decreased after 1 day of adipogenic induction (Figures $1 \mathrm{~g}$ and $\mathrm{h}$ ). Immunofluorescence results showed that little expression of the transcription 
factor HNF1b was expressed in the nucleus of differentiated 3T3-L1 cells (Figure 1i), indicating that inactivation of HNF1b was associated with the adipogenic differentiation of 3T3-L1 cells. The data demonstrated that HNF1b possibly had a negative role in the regulation of adipocyte differentiation.

HNF1b inhibits adipogenic differentiation in 3T3-L1 cells. To examine the exact role of HNF1b in adipogenic differentiation, we knocked down and up the expression of HNF1b in 3T3-L1 preadipocytes using lentivirus (LV) transfection. We detected the mRNA and protein expression of HNF1b to confirm the efficiency of LV transfection (Supplementary Figures $1 \mathrm{~A}$ and B). LV-mediated constant downregulation of HNF1b significantly increased BODIPY and Oil Red $\mathrm{O}$ staining, indicating the increase of basal and rosiglitazone (RSG)-stimulated lipid droplets formation (Figures $2 \mathrm{a}-\mathrm{c}$ ). Flow cytometry analysis also confirmed that knockdown of HNF1b increased the percentage of differentiated adipocytes (Supplementary Figures 2A and B). In contrast, constant upregulation of HNF1b significantly decreased the basal and RSG-stimulated lipid droplets formation (Figures 2d-f). Moreover, we evaluated the effect of transient overexpression of HNF1b on 3T3-L1 adipocyte differentiation using plasmids transfection. The results of real-time PCR and western blot confirmed the efficiency of transient overexpression of HNF1b (Supplementary Figure 1c). Transient overexpression of HNF1b also inhibited basal and RSG-stimulated Oil Red $O$ staining in differentiated 3T3-L1 cells (Figure 2g), indicating the attenuation of lipid droplet formation. These results demonstrated that downregulation of HNF1b was required for the normal adipocyte differentiation.

HNF1b inhibits PPARy and PPARy-dependent gene expression. In the next step, we addressed the possible mechanism of HNF1b-exhibited regulation of adipocyte differentiation. We examined the effect of LV-mediated knockdown of HNF1b on peroxisome proliferator-activated receptor $\gamma$ (PPAR $\gamma)$ and its target gene expression. As shown in Figure 3a, basal mRNA expression of PPARy1, PPARy2, adiponectin, AGPAT2, adipocyte protein 2 (aP2), aquaporin 7 , CD36, lipoprotein lipase (LPL) and perilipin were increased
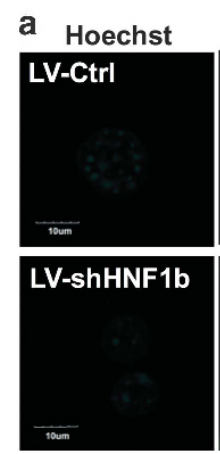

d Hoechst
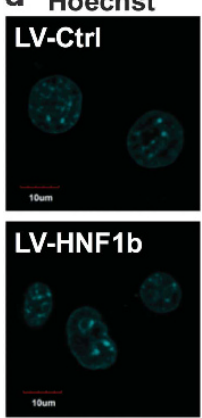

g

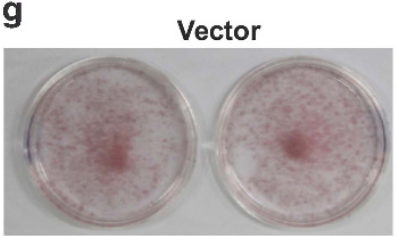

b
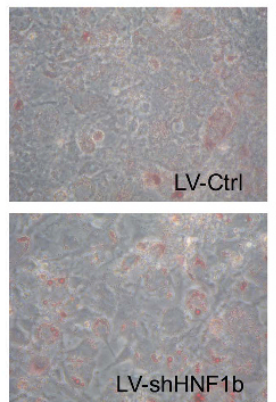

e
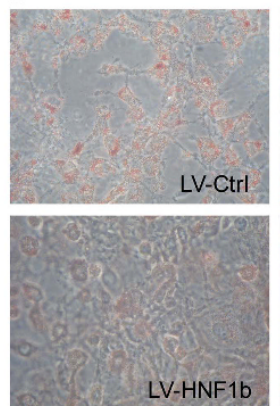

LV-HNF1b
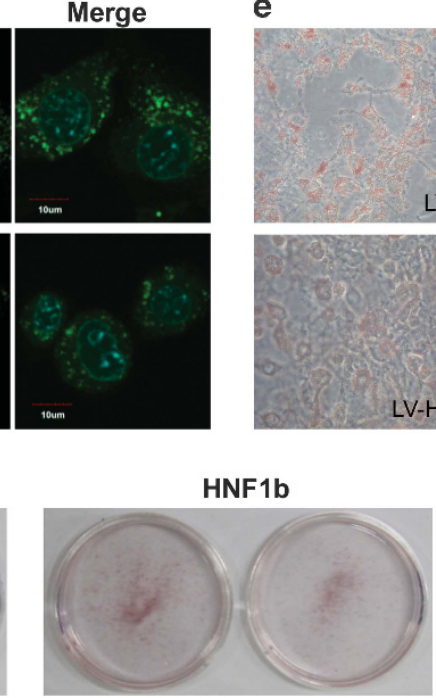

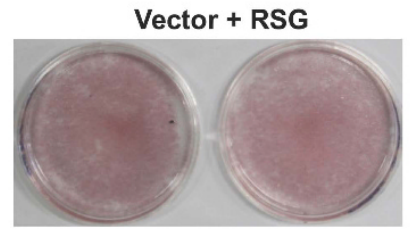

C

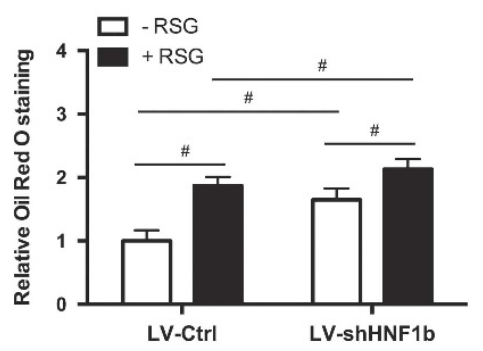

f

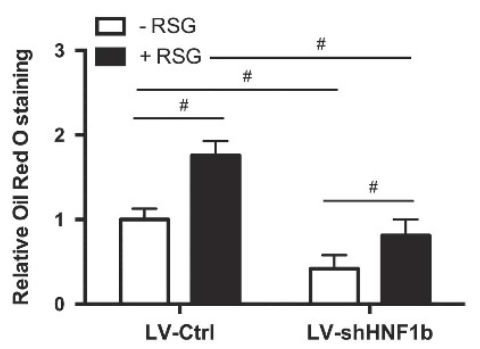

HNF1b + RSG

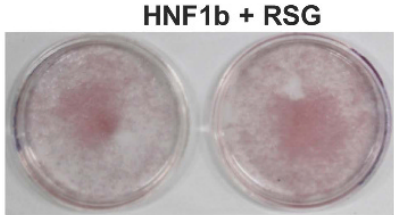

Figure 2 Effect of knockdown and knockup of HNF1b on 3T3-L1 adipocyte differentiation. 3T3-L1 cell lines with stable knockdown (KD) and knockup (KU) of HNF1b were established using lentivirus transfection. HNF1b KD and KU 3T3-L1 cells were induced to adipogenic differentiation. (a and $\mathbf{b})$ BODIPY and Oil Red O staining in differentiated HNF1b KD 3T3-L1 cells with or without $1 \mu$ M RSG treatment. Statistical analysis of Oil Red O staining was shown (c). (d and e) BODIPYand Oil Red O staining in differentiated HNF1b KU 3T3-L1 cells with or without $1 \mu \mathrm{M}$ RSG treatment. Statistical analysis of Oil Red O staining was shown (f). 3T3-L1 cells were transiently transfected with HNF1b plasmid and then induced to adipogenic differentiation. (g) Oil Red O staining in differentiated 3T3-L1 cells transfected with HNF1b plasmid with or without $1 \mu \mathrm{M}$ RSG treatment. ${ }^{\sharp} P<0.05$ 

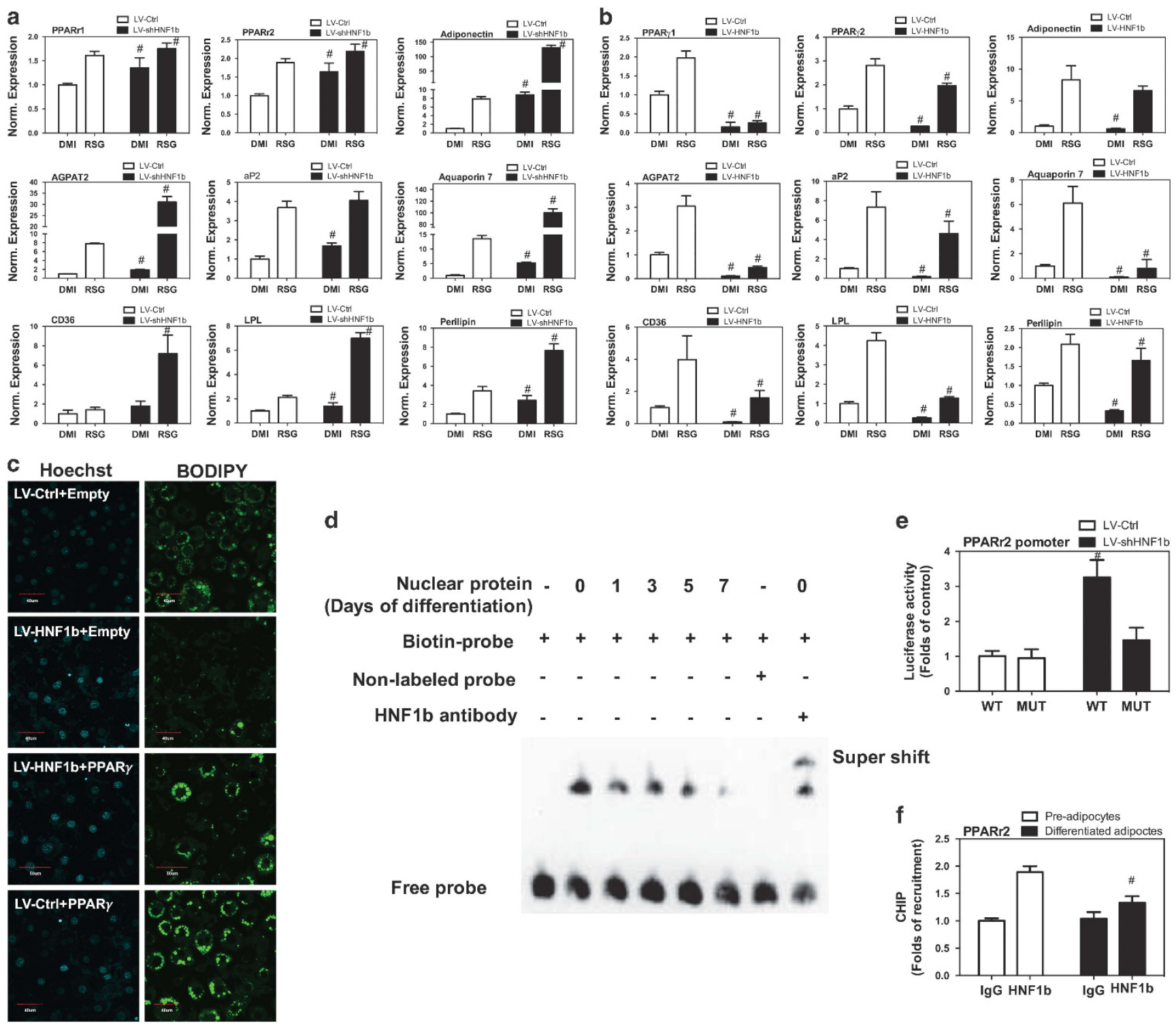

d

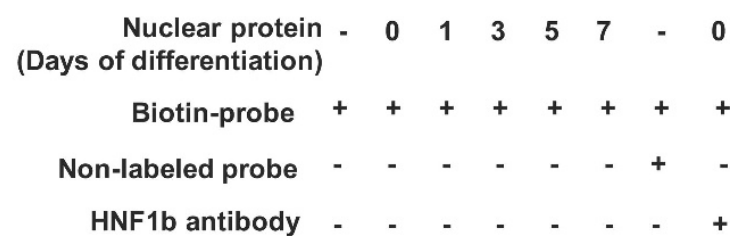

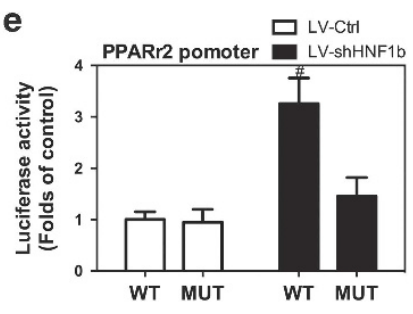

Super shift

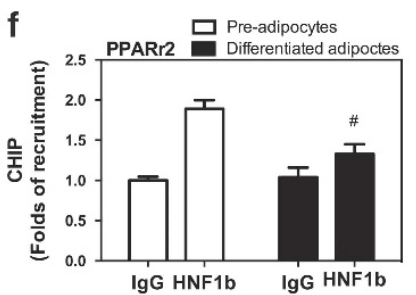

Figure 3 Effect of knockdown and knockup of HNF1b on PPAR $\gamma$ and its target gene expression in differentiated 3T3-L1 adipocytes. 3T3-L1 cell lines with stable KD and KU of HNF1b were established using lentivirus transfection. HNF1b KD and KU 3T3-L1 cells were induced to adipogenic differentiation. (a) mRNA expression of PPAR $\gamma 1$, PPAR $\gamma 2$, adiponectin, AGPAT2, aP2, aquaporin 7, CD36, LPL and perilipin in differentiated HNF1b KD 3T3-L1 cells. (b) mRNA expression of PPAR 1 , PPAR 2 , adiponectin, AGPAT2, aP2, aquaporin 7, CD36, LPL and perilipin in differentiated HNF1b KU 3T3-L1 cells. HNF1b KU 3T3-L1 cells were transfected with PPAR $\gamma$ plasmid and induced to adipogenic differentiation. (c) BODIPY staining in differentiated HNF1b KU 3T3-L1 cells transfected with PPAR $\gamma$ plasmid. (e) 3T3-L1 preadipocytes were induced to adipogenic differentiation. On days $0,1,3,5$ and 7 , nuclear extracts were obtained and EMSA detection of HNF1b-binding in the putative binding sites in the PPAR 2 promoter was performed. (d) 3T3-L1 cell lines with stable KD and KU of HNF1b were established using lentivirus transfection. The cells were transfected with wild-type promoter (WT) or its derivative containing site-direct mutagenesis of the putative HNF1b-binding site (MUT). Fold change of luciferase activity was calculated. (f) 3T3-L1 preadipocytes were induced to adipogenic differentiation and HNF1b-binding of PPAR $\gamma$ promoter in preadipocytes and differentiated adipocytes were determined. ${ }^{*} P<0.05$, compared with respective control

by knockdown of HNF1b in differentiated 3T3-L1 adipocytes. Knockdown of HNF1b also promoted RSG-stimulated mRNA expression of PPARy1, PPARy2, adiponectin, AGPAT2, aP2, aquaporin 7, CD36, LPL and perilipin in differentiated 3T3-L1 adipocytes (Figure $3 a$ ). In contrast, overexpression of HNF1b significantly decreased basal and RSG-stimulated mRNA expression of these genes in differentiated 3T3-L1 adipocytes (Figure $3 b$ ). To validate the role of PPARy in HNF1bexhibited regulation of adpogenic differentiation, 3T3-L1 cells carrying LV-HNF1b were transfected with PPARy plasmids and induced to adipogenic differentiation. We showed that upregulation of PPARY significantly suppressed LV-HNF1bmediated inhibition of BODIPY staining (Figure $3 c$ ), indicating a role of reduction of PPARy in HNF1b-exhibited inhibition of lipid droplet formation. To provide direct evidence that HNF1b binds to the PPAR 2 promoter, the double-stranded oligonucleotides of the putative HNF1b-binding element were biotinlabeled and used as probes for EMSA. Incubation of nuclear protein extracts with the probe resulted in the formation of major shifted bands (DNA-protein complex; Figure 3d), 
which was attenuated as the differentiation prolonged. Moreover, the shifted band was competed by unlabeled probe and supershifted by HNF1b antibody (Figure 3d). To provide direct evidence that the HNF1b-binding site within the PPARY2 promoter actually contributed to HNF1b-induced regulation of PPAR 2 transcription, we performed site-directed mutagenesis of the HNF1b-binding site in pGL3 luciferase driven by the PPARY2 promoter. A base pair change from TT (at positions -688 and -687) to GC in this HNF1b-binding element significantly suppressed PPARy2 promoter repression by HNF1b (Figure $3 e$ ). These results suggested that the HNF1b-binding site within the PPARy2 promoter was required for HNF1b-induced regulation of PPARy2 transcription. To further validate the regulation of PPARy expression by HNF1b in a cell, we detected PPARy2 promoter expression after HNF1b immunoprecipitation. As shown in Figure 3f, in undifferentiated 3T3-L1 cells, we detected a significant binding of HNF1b in the promoter of PPARy2. In differentiated 3T3-L1 adipocytes, HNF1b-exhibited binding in the promoter of PPARy2 was notably inhibited (Figure 3f). Collectively, these results provide evidence that HNF1b repressed PPARY2 and its target gene expression through direct binding to the PPARy2 promoter.
HNF1b induces apoptotic and autophagic cell death. The effect of dysregulation of HNF1b on apoptosis and autophagy was evaluated. In Figures $4 \mathrm{a}$ and $\mathrm{b}$, we showed that knockdown of HNF1b significantly decreased the percentage of TUNEL-stained cells on differentiation day 2, indicating the attenuation of apoptosis. In contrast, knockup of HNF1b markedly increased the number of apoptotic cell, as evidenced by increase of TUNEL-positive 3T3-L1 adipocytes (Figures 4c and d). In addition, knockdown of HNF1b increased the mRNA expression of cdc25, cyclin $A$ and cyclin D1, but decreased the mRNA expression of cyclin E, p21, p53 and p57 (Supplementary Figure 3A). Knockup of HNF1b decreased the mRNA expression of cdc25, CDK6, cyclin A, cyclin B, cyclin D1 and cyclin E, but increased the mRNA expression of p21, p53 and p57 (Supplementary Figure $3 \mathrm{~B}$ ). The results indicated that dysregulation of HNF1b could influence cell cycle progress and apoptosis through altering the expression of cell cycle checkpoints and apoptotic regulators. Moreover, we found that upregulation of HNF1b promoted the formation of autophagosome in 3T3-L1 adipocytes (Figure 4e), indicating the enhancement of autophagy. To test whether the regulation of PPARy was involved in HNF1b-induced cell death, LV-HNF1b cells were transfected with PPARy plasmids. We showed that upregulation of PPARy did not significantly affect HNF1b-induced
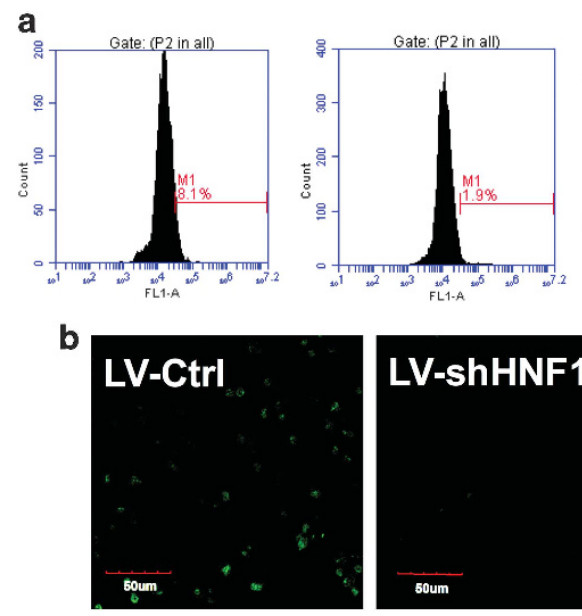
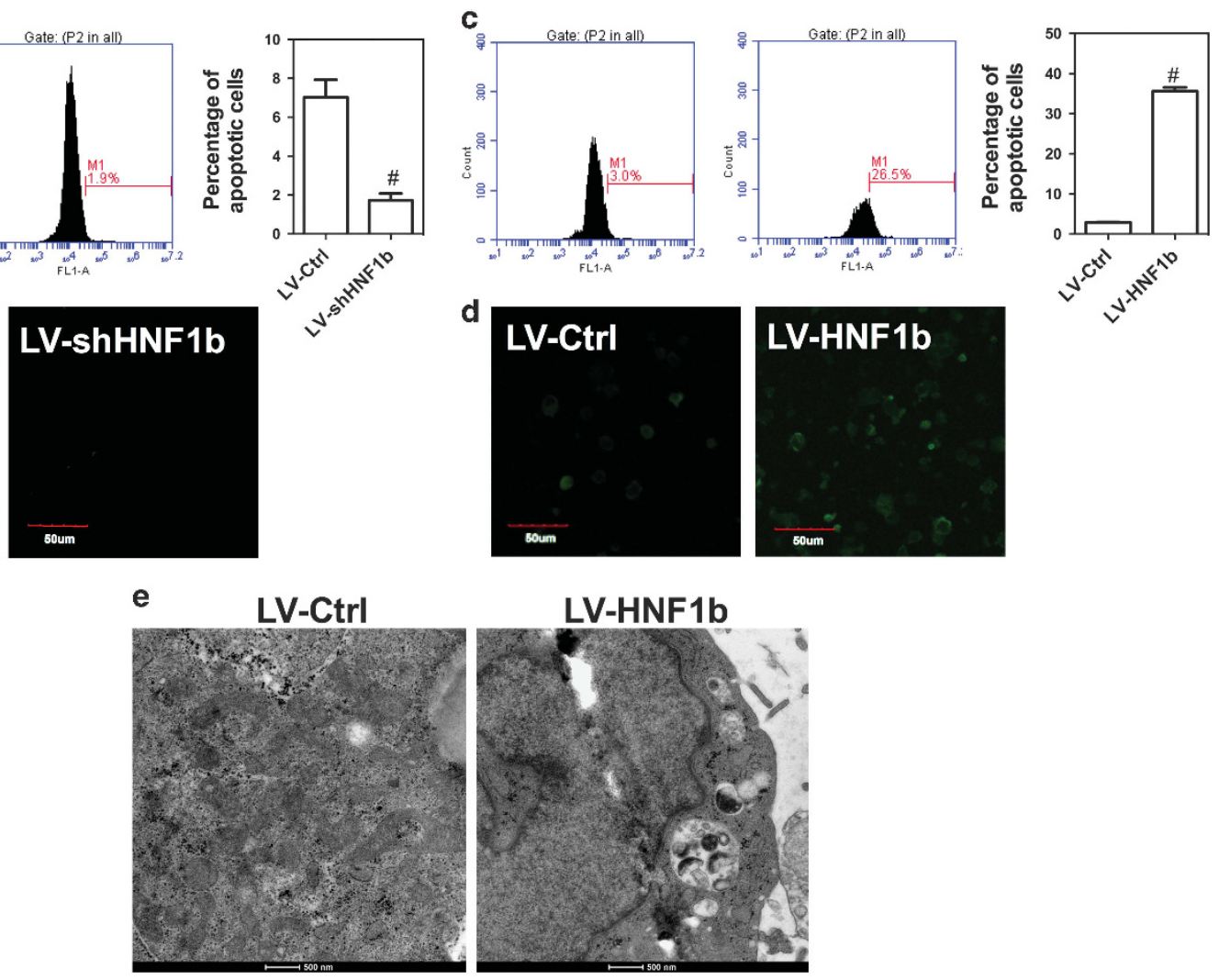

Figure 4 Effect of knockdown and knockup of HNF1b on apoptosis and autophagy in differentiated 3T3-L1 adipocytes. 3T3-L1 cell lines with stable KD and KU of HNF1b were established using lentivirus transfection. HNF1b KD and KU 3T3-L1 cells were induced to adipogenic differentiation. (a) Flow cytometry analysis of TUNEL-stained cells in differentiated HNF1b KD 3T3-L1 cells. (b) Confocal observation of TUNEL-stained cells in differentiated HNF1b KD 3T3-L1 cells. (c) Flow cytometry analysis of TUNEL-stained cells in differentiated HNF1b KU 3T3-L1 cells. (d) Confocal observation of TUNEL-stained cells in differentiated HNF1b KU 3T3-L1 cells. (e) Images of differentiated HNF1b KD 3T3-L1 cells under TEM. ${ }^{\#} P<0.05$, compared with control 

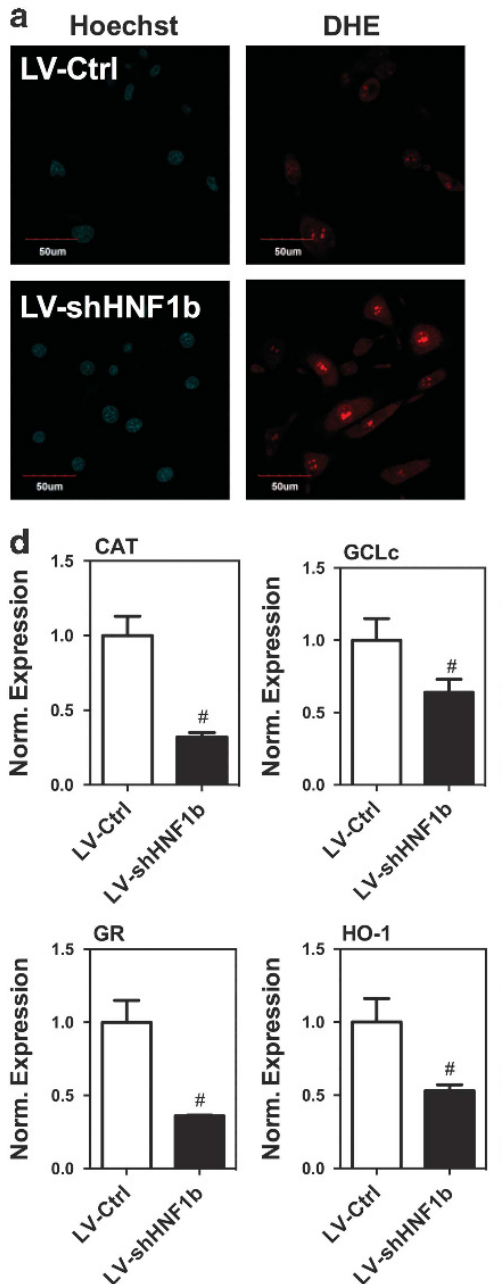
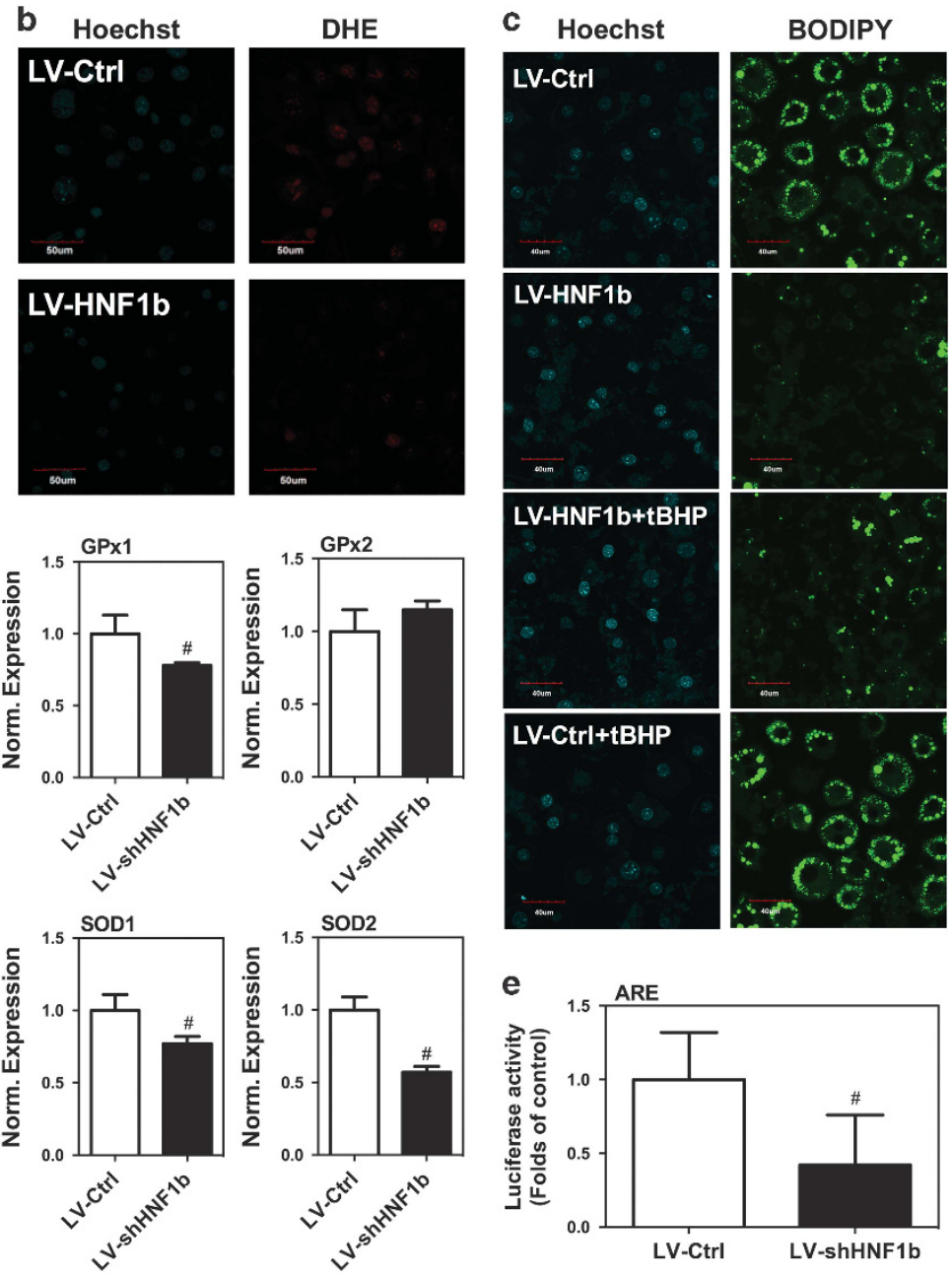

Figure 5 Effect of knockdown and knockup of HNF1b on redox state in differentiated 3T3-L1 adipocytes. 3T3-L1 cell lines with stable KD and KU of HNF1b were established using lentivirus transfection. HNF1b KD and KU 3T3-L1 cells were induced to adipogenic differentiation. (a) DHE staining in differentiated HNF1b KD 3T3-L1 cells. (b) DHE staining in differentiated HNF1b KU 3T3-L1 cells. HNF1b KU 3T3-L1 cells were treated by tBHP and induced to adipogenic differentiation. (c) BODIPY staining in differentiated HNF1b KU 3T3-L1 cells treated by tBHP. (d) mRNA expression of CAT, GCLc, GPx1, GPx2, GR, HO-1, SOD1 and SOD2 in differentiated HNF1b KD 3T3-L1 cells. HNF1b KD 3T3-L1 cells were transfected with pARE-luc and ARE luciferase activity was examined. ${ }^{\#} P<0.05$, compared with control

reduction of cell viability (Supplementary Figure $3 \mathrm{C}$ ) and increase of apoptosis (Supplementary Figure 3D). These results demonstrated that overexpression of $\mathrm{HNF} 1 \mathrm{~b}$ induces apoptotic and autophagic cell death, which contributed to the reduction of adipocyte differentiation.

HNF1b regulates ROS homeostasis through regulation of antioxidant enzymes. Next, we examined the effect of dysregulation of HNF1b on ROS level in 3T3-L1 cells. In Figure $5 \mathrm{a}$ and Supplementary Figure $4 \mathrm{~A}$, we showed that knockdown of HNF1b significantly decreased DHE fluorescence intensity and DHE-positive 3T3-L1 cells, indicating the increase of ROS level. In contrast, knockup of HNF1b induced a significant decrease in ROS level (Figure 5b). To test the role of changes of ROS level in LV-HNF1b-mediated inhibition of adipocyte differentiation, LV-HNF1b 3T3-L1 cells were exposed to tert-Butyl hydroperoxide (tBHP), an analog of hydrogen peroxide, in the process of adipogenic differentiation. tBHP treatment suppressed the decrease of droplet formation in differentiated 3T3-L1 adipocytes induced by LV-HNF1b (Figure 5c), indicating the changes of ROS level had a role in the dysregulation of HNF1b-mediated modulation of adipocyte differentiation. We tested whether the regulation of PPARy was involved in HNF1b-induced alteration of ROS level and found that upregulation of PPARY did not significantly affect HNF1b-induced reduction of ROS level (Supplementary Figure 4B). We further determined the effect of knockdown of HNF1b on the expression of a battery of antioxidant enzymes. Knockdown of HNF1b significantly decreased the mRNA expression in catalase (CAT), glutamate-cysteine ligase catalytic subunit (GCLc), glutathione peroxidase 1 (GPx1), glutathione reductase $(G R)$, heme oxygenase-1 (HO-1), superoxide dismutase 1 (SOD1) and SOD2 (Figure 5d). Moreover, we transfected 3T3-L1 cells with antioxidant response element (ARE) luciferase plasmids and tested the direct transcriptional regulation of antioxidant 
enzymes by HNF1b. We showed that knockdown of HNF1b resulted in a significant reduction in ARE luciferase activity, indicating that HNF1b had an antioxidant role through transcriptional regulation of the antioxidant enzymes (Figure 5e). The result demonstrated HNF1b could influence adipocyte differentiation through transcriptional regulation of ROS homeostasis.

\section{Discussion}

Although a large number of literatures have suggested a substantial role of mutation of HNF1b gene in the pathogenesis of MODY $5,{ }^{20-23}$ the role of HNF1b in the regulation of glucose and lipid metabolism is unclear. A study found that shRNA-mediated reduction of HNF1b in liver caused glucose intolerance, impaired insulin signaling and promoted hepatic gluconeogenesis. ${ }^{24}$ In turn, the authors also found that hepatic overexpression of HNF $1 b$ improved insulin sensitivity in $\mathrm{db} / \mathrm{db}$ mice, ${ }^{24}$ suggesting a critical role of HNF1b in the protection against insulin resistance and glucose metabolic dysfunction. Obesity and abnormal accumulation of fat have a fundamental role in the development of various metabolic diseases. ${ }^{25-28}$ Obesity is defined as an excessive accumulation of fat mass in WAT to the extent that health may be adversely affected, which can occur through an increase of adipocyte volume (hypertrophy), number (hyperplasia) or a combined form (hypertrophy-hyperplasia). ${ }^{29,30}$ Therefore, the differentiation of adipocytes is of great importance for the physiological and pathophysiological function of adipose tissue. However, the role of HNF1b in adipocyte differentiation is unknown.

In the current study, we examined the exact role of HNF1b in the regulation of adipocyte differentiation. We found that the expression of HNF1b was relatively lower in WAT, compared with that in the liver, kidney, pancreas and so on. In WAT of both diet-induced and genetic obese mice, the expression of $\mathrm{HNF} 1 \mathrm{~b}$ was reduced, indicating a negative role of HNF1b in adipose formation. Moreover, the expression of HNF1b decreased during the process of adipocyte differentiation in vitro. Furthermore, we examined the role of HNF1b in the regulation of adipocyte differentiation. We found that stable downregulation of HNF1b enhanced 3T3-L1 adipocyte differentiation, both stable and transient upregulation of HNF1b inhibited 3T3-L1 adipocyte differentiation, indicating a negative role of HNF1b in the process of adipocyte differentiation.

Adipocyte differentiation, a complex and programmed process from multipotent stem cells or preadipocyte precursors to mature adipocytes, has a pivotal role in the formation of lipid droplet and fat mass. ${ }^{31-33}$ Briefly, the complicated process is divided into four steps: initial growth arrest, mitotic clonal expansion (MCE), early differentiation and terminal differentiation-final formation of mature adipocyte phenotype. ${ }^{34}$ The process of adipocyte differentiation is sequentially and coordinately regulated by a series of transcription factors which modulate the expression of hundreds of genes responsible for the establishment of the mature fat-cell phenotype. ${ }^{31,32,33}$ Among those transcription factors, PPARy is the central regulator responsible for adipogenesis. ${ }^{32,34}$ PPARy is the target of antidiabetic drugs thiazolidinediones ${ }^{35}$ and is essential for animals to develop adipose tissue. ${ }^{36}$ PPARy regulates adipocyte differentiation through transcriptionally modulating multiple adipocytespecific genes, ${ }^{37}$ including adiponectin, AGPAT2, aP2, aquaporin 7, CD36, LPL and perilipin and so on. ${ }^{38}$ PPARy is expressed as two isoforms, PPARy1 and PPARy2. PPARy1 is expressed in many tissues, whereas PPARy2 expression is restricted almost exclusively to adipose.

To investigate the mechanism of HNF1b-exhibited regulation of adipocyte differentiation, possible regulation of PPARy by HNF1b was tested. The results showed that upregulation of HNF1b inhibited PPARy and its target gene expression, but downregulation of HNF1b increased those genes expression. Moreover, overexpression of PPARy suppressed HNF1b knockup-induced inhibition of adipocyte differentiation. HNF1b inhibited the transcription of PPARy2 through direct binding in the promoters. The results suggested that HNF1b functioned as a transcriptional repressor in the regulation of adipocyte differentiation.

MCE is a prerequisite for terminal differentiation of adipocytes. ${ }^{39}$ In the early process of adipocyte differentiation, in response to adipogenic stimuli, growth-arrested 3T3-L1 preadipocytes synchronously re-enter the cell cycle and undergo MCE followed by sequential expression of adipocyte phenotype-specific genes. ${ }^{39}$ Cell proliferation is rapid during MCE and cell death is harmful to normal MCE. We also examined the effect of HNF1b on MCE during 3T3-L1 adipocyte differentiation. HNF1b promoted apoptotic and autophagic cell death in early differentiated adipocytes through regulation of cell cycle progress and cell deathrelated factors. The results suggested that induction of cell death and thus the inhibition of MCE was involved in the inhibitory effect of HNF1b in adipocyte differentiation.

Redox state is closely associated with fat accumulation and lipid metabolism. In obesity, fat mass is associated with systemic oxidative stress in both humans and animals. ${ }^{40}$ Mitochondria-generated ROS has been found to enhance adipocyte differentiation. ${ }^{41}$ Moreover, ROS could promote adipocyte differentiation through accelerating MCE. ${ }^{42}$ In our study, we also found that HNF1b acted as an antioxidant regulator through regulating various antioxidant enzymes via binding with ARE. Oxidant treatment suppressed LV-HNF1binduced inhibition of adipocyte differentiation, suggesting that the antioxidant role of HNF1b was involved in its negative regulation of adipocyte differentiation.

A previous study has reported that there was no significant changes of fat mass in heterozygous HNF1b-deficient mice $(\mathrm{HNF} 1 \mathrm{~b}+/-)$ and adenoviruses-shHNF1b-mediated reduction of hepatic HNF1b protein expression had no effect on body weight, fat mass, adipocyte size or WAT inflammation. ${ }^{24}$ Adipose-specific HNF1b-knockout mice are needed for further study of HNF1b function in the regulation of adipose formation in vivo. Overall, our results suggest that HNF1b is novel negative regulator of fat accumulation and adipocyte differentiation through regulation of PPARy signaling, MCE and redox state (Figure 6). Considering the significant role of adipocyte differentiation in the formation and function of adipose, clarification of the mechanism of HNF1b-mediated regulation of adipocyte differentiation is important for the exploration of strategies for the treatment of metabolic disorders. In combination with previous findings of the beneficial role of HNF1b against insulin resistance and 


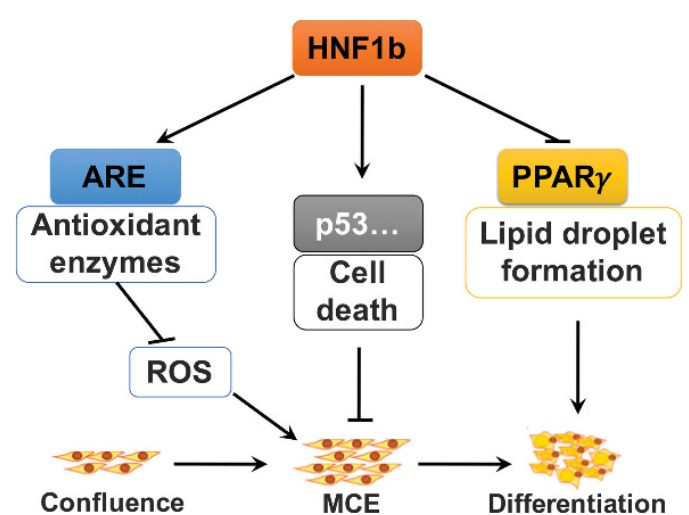

Figure 6 Schematic figure of the molecular mechanisms underlying inhibitory role of HNF1b in adipocyte differentiation. HNF1b has an inhibitory role in adipocyte differentiation and adipose formation. The mechanism involves downregulation of ROS level, promotion of cell death, and downregulation of PPAR $\gamma$ and its target gene expression, leading to inhibition of adipocyte differentiation

diabetes, we proposed that HNF1b may be a crucial therapeutic target for the treatment of obesity, diabetes and related diseases.

\section{Materials and Methods}

Materials. The $\beta$-actin antibody and IBMX were purchased from Santa Cruz Biotechnology (Santa Cruz, CA, USA). HNF1b antibody was obtained from ProSci Technology (Poway, CA, USA). Hoechst and DHE were purchased from Beyotime Institute of Biotechnology (Jiangsu, China). BODIPY were purchased from Invitrogen (Carlsbad, CA, USA). DAPI was purchased from Bioworld Technology (Bioworld Technology, Co., Ltd, Nanjing, China). Oil Red O, insulin, tBHP and most of the chemicals and reagents used in this study were procured from Sigma (St. Louis, MO, USA).

Animals. All the experiments were performed according to the procedures approved by the Fourth Military Medical University Animal Care and Use Committee. The SD rats and C57 mice were purchased from Animal Centre of Fourth Military Medical University. The tissues of SD rats and C57 mice were obtained to evaluate the tissue-specific distribution of HNF1b. Wild-type and C57BL/KsJ-db/db obese diabetic mice were purchased from Model Animal Research Center of Nanjing University. The C57 mice were administrated with high-fat (HF) diet for 3 months to induce obesity. Epididymal adipose tissue was collected to detect HNF1b expression level. The animals were housed under temperature $\left(23 \pm 2{ }^{\circ} \mathrm{C}\right)$ and humidity $(55 \pm 5 \%)$ conditions with a standard light (12 h light/dark) cycle.

Cell culture and treatment. The 3T3-L1 cells were cultured and induced to adipogenic differentiation as we previously did. ${ }^{43}$ Briefly, 3 T3-L1 preadipocytes were cultured in DMEM containing $10 \%$ fetal calf serum (FCS) until the cells became confluent. Two days post confluence (Day 0), the cells were induced to adipogenic differentiation using DMI differentiation medium consisting of DMEM containing $10 \%$ fetal bovine serum (FBS), $0.5 \mathrm{mM}$ isobutylmethylxanthine (IBMX), $1 \mu \mathrm{M}$ dexamethasone (DEX) and $167 \mathrm{nM}$ insulin for 2 days. At day 2, the cells were then maintained in a 10\% FBS/DMEM medium with $167 \mathrm{nM}$ insulin for another 2 days (Day 4) and then cultured in 10\% FBS/DMEM medium for an additional 4 days (Day 8). At the end, more than $90 \%$ of cells became mature adipocytes with lipid-filled droplets. In some experiments, the induction medium contained $1 \mu \mathrm{M}$ rosiglitazone (RSG). All media contained penicillin (100 U/ml), streptomycin $(100 \mu \mathrm{g} / \mathrm{ml})$ and glutamine $(292 \mu \mathrm{g} / \mathrm{l})$. The cells were maintained at $37^{\circ} \mathrm{C}$ in a humidified $5 \% \mathrm{CO}_{2}$ atmosphere.

Transfection of plasmids. A total $20-30 \%$ confluent $3 \mathrm{~T} 3-\mathrm{L} 1$ preadipocytes were transfected with HNF1b lentivirus (LV), HNF1b short hairpin RNA (shRNA) LV or control LV according to the manufacturer's protocols (GeneChem, Shanghai, China). Forty-eight hours after the transfection, the cells were purified with $5 \mu \mathrm{g} / \mathrm{ml}$ puromycin overnight. The 3T3-L1 cell lines with stable knockup (KU) and knockdown (KD) were established. The 3T3-L1 preadipocytes were also transiently transfected with HNF1b plasmids (GeneChem, Shanghai, China) or control empty plasmids. HNF1b KU 3T3-L1 cells were transiently transfected with the PPAR $\gamma$ plasmids (Addgene, Cambridge, MA, USA) or empty vectors. Briefly, serum-free DMEM medium containing $4 \mu \mathrm{g}$ p-HNF1b or pPPAR $\gamma$ or empty plasmids was mixed with $6 \mu$ l Turbofect Transfection Reagents (Thermo Fisher Scientific, Waltham, MA, USA) and added to the cells. After 4-6 h, the transfection medium was removed and the cells were incubated with DMEM medium containing $10 \%$ FBS. Forty-eight to 96 hours after the transfection, plasmids transfection efficiency was evaluated.

Determination of lipid accumulation. The differentiated cells were fixed with $4 \%$ paraformaldehyde for $30 \mathrm{~min}$ and then stained with $3.5 \mathrm{ng} / \mathrm{ml}$ BODIPY (3922; room temperature, $20 \mathrm{~min}$ ) to detect lipid droplet. The images were captured using a confocal microscope (Olympus, FV10i, Tokyo, Japan). Otherwise, the cells were fixed with $4 \%$ paraformaldehyde for $30 \mathrm{~min}$, rinsed with water and then stained with Oil Red $\mathrm{O}$ solution (six parts of saturated Oil Red $\mathrm{O}$ dye in isopropanol plus four parts of water) for $30 \mathrm{~min}$. The cells were washed with water to remove excessive dye. Lipid accumulation in cells was observed under a light microscope (Olympus BX51 with DP70 camera).

Real-time PCR. Total RNA was isolated from tissues and cells using RNA Isolation Assay Kit (TIANGEN, Beijing, China). The concentration of RNA was determined and $500 \mathrm{ng}$ of RNA was used to reversely transcribed into cDNA using TaKaRa PrimeScript RT-PCR Kit (TaKaRa, Dalian, China). One microliter cDNA was used to perform real-time PCR in $20 \mu$ l reaction mixtures with SYBR Green PCR kit (Thermo Fisher Scientific, Rockford, IL, USA) using a CFX96 real-time PCR system (Bio-Rad, Hercules, CA, USA). The PCR reaction conditions were as follows: initial denaturation at $95^{\circ} \mathrm{C}$ for $10 \mathrm{~min}$ followed by 30 cycles at $95^{\circ} \mathrm{C}$ for $1 \mathrm{~min}$, annealing at $53^{\circ} \mathrm{C}$ for $1 \mathrm{~min}$, extension at $72^{\circ} \mathrm{C}$ for $1 \mathrm{~min}$ and final extension at $72{ }^{\circ} \mathrm{C}$ for $5 \mathrm{~min}$. The relative amount of RNA was quantified using the comparative cycle threshold $\left(C_{T}\right)$ $\left(2^{-\Delta \Delta} C_{T}\right)$ method. $\beta$-Actin was used as internal controls. The results were expressed as folds of control. The primers used were shown in Supplementary Table 1.

Western blot. Protein extractions of tissues and cells were separated using RIPA Iysis buffer (TIANGEN). Protein concentration was determined using BCA method (Thermo Fisher Scientific, Rockford, IL, USA). Equal volumes of protein extraction and loading buffer were mixed and separated using SDS-PAGE on $10 \%$ polyacrylamide gels. The separated samples were transferred onto nitrocellulose (NC) membranes. After blocking in $8 \%$ fat-free milk for $1 \mathrm{~h}$ at room temperature, the membranes were incubated with primary antibodies at $4{ }^{\circ} \mathrm{C}$ overnight. After washing four times, the membranes were incubated in the appropriate HRP-conjugated secondary antibody at $37^{\circ} \mathrm{C}$ for $30 \mathrm{~min}$. Protein bands were detected using chemiluminescent reagents according to the manufacturer's protocol and observed using an image analyzer Quantity One System (Bio-Rad). The protein quantifications were adjusted for the corresponding $\beta$-actin level.

ROS determination. ROS was detected by specific probe DHE as we previously described. ${ }^{44,45}$ Briefly, the cells in specific dishes were stained with $10 \mu \mathrm{M}$ DHE in serum-free DMEM medium (kept in the dark, $37^{\circ} \mathrm{C}$ ) for $20 \mathrm{~min}$. After washing twice with PBS, the cells were observed under a laser scanning confocal microscope (Olympus, FV10i). Otherwise, $1 \times 10^{6} \mathrm{cell} / \mathrm{s} / \mathrm{ml}$ suspension was added to $10 \mu \mathrm{M}$ DHE and incubated in the incubator at $37^{\circ} \mathrm{C}$ for $30 \mathrm{~min}$. ROS level was determined using flow cytometry (BD, C6, San Jose, CA, USA) analysis.

Chromatin immunoprecipitations. Chromatin immunoprecipitations (CHIP) were conducted using a Thermo Scientific Pierce Agarose ChIP Kit according to the manufacture's instruction (Thermo Fisher Scientific, Rockford, IL, USA). After the treatment, the cells were cross-linked using formaldehyde (1\%). Then, the cross-linked cells were collected, lysed in SDS lysis buffer, and sheared by sonication. The sonicated cell lysate was immunoprecipitated using antibody against HNF1b. The immune complexes were recovered by adding protein A-agarose. After washing the DNA-protein complexes with saline, DNA was extracted with phenol/chloroform, precipitated, redissolved and used as templates for real-time PCR with the following primer. The primers used to amplify PPAR $\gamma$ promoter was: 5'-TTCAGATGTGTGATTAGGAG-3' and 5'-AGACTTGGTACATTAC AAGG-3' as previously reported. ${ }^{46}$

Promoter reporters and luaciferase assay. The full-length PPAR $\gamma$ promoter was subcloned into the pGL3-Basic firefly luciferase reporter (Promega, Beijing, China; pPPAR $\gamma$-Luc). The mutant constructs were generated by using the 
QuickChange Site-Directed Mutagenesis kit (Stratagene, La Jolla, CA, USA). A base pair change from TT (at positions -688 and -687 ) to $\mathrm{GC}$ was constructed. HNF1b KD 3T3-L1 cells were transfected with Renilla TK (5 ng, Promega) and pARE-luc (100 ng) or WT or MUT pPPAR $\gamma$-Luc (100 ng) plasmids using Turbofect Transfection Reagent. The cells were washed with cold PBS, and lysed in $200 \mu$ l of lysis buffer. The cell extracts were cleared by centrifugation at 12000 r.p.m. for $2 \mathrm{~min}$ at $4{ }^{\circ} \mathrm{C}$. The supernatants were assayed for luciferase activity using dualluciferase reporter assay system (Promega). Firefly luciferase activity was normalized to Renilla luciferase and expressed as a ratio of relative light units.

EMSA and supershift assay. Nuclear protein extracts were prepared and protein concentrations were determined using the BCA method (Thermo Fisher Scientific, Rockford, IL, USA), according to the manufacturer's protocol. Biotin labeling of probes was performed using the biotin $3^{\prime}$-end DNA-labeling kit (Thermo Fisher Scientific, Rockford, IL, USA) according to the manufacturer's instructions. Single-stranded oligos were labeled individually and annealed for $1 \mathrm{~h}$ at room temperature to produce double-stranded probes. A $3 \mu \mathrm{g}$ weight of the nuclear protein was incubated at room temperature with the binding buffer (Thermo Fisher Scientific, Rockford, IL, USA) for $20 \mathrm{~min}$. Then biotin 3 '-end-labeled DNA probes $\left(5^{\prime}\right.$-TCAATAATTCAT- $\left.3^{\prime}\right)$ were added. For competition or antibody supershift, the respective unlabeled DNA probes (100-fold excess) or antibody were added to the reaction mixture before addition of labeled probes. Free probe and protein-DNA complexes were separated on $6 \%$ neutral polyacrylamide gel and transferred to a nylon membrane for detection using the Lightshift electrophoretic mobility shift assay kit (Thermo Fisher Scientific, Rockford, IL, USA).

Apoptosis. Apoptosis was determined using In Situ Cell Death Detection Kit (Roche, Basel, Switzerland). Briefly, the cells in specific dishes were fixed using paraformaldehyde and stained with Label Solution solution for $20 \mathrm{~min}$. After washing twice with PBS, the cells were observed under a laser scanning confocal microscope (Olympus, FV10i). Otherwise, cell suspension was added to the TUNEL reaction mixture that contains TdT and fluorescein-dUTP. After washing twice with PBS, ROS level was determined using flow cytometry (BD, C6) analysis.

Immunofluorescence. The cells were fixed in a freshly prepared solution of $4 \%$ paraformaldehyde, rinsed and permeabilized with $0.1 \%$ Triton X-100 in PBS. Nonspecific binding was blocked and the cells were incubated with anti-HNF1b antibody overnight at $4{ }^{\circ} \mathrm{C}$ and then incubated with Cy3-conjugated antibodies. BODIPY was used to label lipid droplet. DAPI was used to label nuclear DNA. Immunofluorescence was examined with a confocal microscope (Olympus, FV10i).

Transmission electron microscopy. Transmission electron microscopy (TEM) assay was performed as previously described. ${ }^{47}$ Briefly, the cells were fixed in $2 \%$ glutaraldehyde in $0.1 \mathrm{M} \mathrm{PBS}$ for $2 \mathrm{~h}$ at $4{ }^{\circ} \mathrm{C}$. Subsequently, they were post-fixed in $1 \%$ osmium tetroxide in $0.1 \mathrm{M}$ PBS for $2 \mathrm{~h}$ at $4{ }^{\circ} \mathrm{C}$. After washing, the sections were dehydrated in a series of water/ethanol mixtures to $100 \%$ ethanol and then infiltrated in sequentially increasing concentrations of araldite $618-100 \%$, embedded in Beam capsules, and placed in a $60{ }^{\circ} \mathrm{C}$ oven for $48 \mathrm{~h}$. The sections were stained with lead citrate and viewed with transmission electron microscope (JEM-2000EX, Japan).

Statistical analysis. Results were expressed as the means \pm SEM. Statistical analysis was carried out sing Graph-Pad Prism software. Significance between more than two groups was analyzed using One-way analysis of variance (ANOVA) followed by the Newmane Keuls multiple-comparison post hoc test. Significance of differences between two groups was analyzed by Student's $t$ test. Data were considered statistically significant for $P<0.05$.

\section{Conflict of Interest}

The authors declare no conflict of interest.

Acknowledgements. This work was supported by National Natural Science Foundation of China (No. 31400724 and No. 21677176).

1. Tronche F, Yaniv M. HNF1, a homeoprotein member of the hepatic transcription regulatory network. Bioessays 1992; 14: 579-587.
2. Cereghini S, Blumenfeld M, Yaniv M. A liver-specific factor essential for albumin transcription differs between differentiated and dedifferentiated rat hepatoma cells. Genes Dev 1988; 2: 957-974.

3. Rey-Campos J, Chouard T, Yaniv M, Cereghini S. vHNF1 is a homeoprotein that activates transcription and forms heterodimers with HNF1. EMBO J 1991; 10: 1445-1457.

4. Ulinski T, Bensman A, Lescure S. Abnormalities of hepatocyte nuclear factor (HNF)-1beta: biological mechanisms, phenotypes, and clinical consequences. Arch Pediatr 2009; 16: 1049-1056.

5. Bell Gl, Xiang KS, Newman MV, Wu SH, Wright LG, Fajans SS et al. Gene for non-insulin-dependent diabetes mellitus (maturity-onset diabetes of the young subtype) is linked to DNA polymorphism on human chromosome 20q. Proc Natl Acad Sci USA 1991; 88: 1484-1488.

6. Edghill EL, Oram RA, Owens M, Stals KL, Harries LW, Hattersley AT et al. Hepatocyte nuclear factor-1beta gene deletions-a common cause of renal disease. Nephrol Dial Transplant 2008; 23: 627-635

7. Edghill EL, Bingham C, Ellard S, Hattersley AT. Mutations in hepatocyte nuclear factor-1beta and their related phenotypes. J Med Genet 2006; 43: 84-90.

8. Bingham C, Hattersley AT. Renal cysts and diabetes syndrome resulting from mutations in hepatocyte nuclear factor-1beta. Nephrol Dial Transplant 2004; 19: 2703-2708.

9. Bellanne-Chantelot C, Clauin S, Chauveau D, Collin P, Daumont M, Douillard C et al. Large genomic rearrangements in the hepatocyte nuclear factor-1beta (TCF2) gene are the most frequent cause of maturity-onset diabetes of the young type 5. Diabetes 2005; 54: $3126-3132$.

10. Han $X$, Luo $Y$, Ren $Q$, Zhang $X$, Wang $F$, Sun $X$ et al. Implication of genetic variants near SLC30A8, HHEX, CDKAL1, CDKN2A/B, IGF2BP2, FTO, TCF2, KCNQ1, and WFS1 in type 2 diabetes in a Chinese population. BMC Med Genet 2010; 11: 81

11. Lauenborg J, Grarup N, Damm P, Borch-Johnsen K, Jorgensen T, Pedersen $O$ et al. Common type 2 diabetes risk gene variants associate with gestational diabetes. J Clin Endocrinol Metab 2009; 94: 145-150.

12. Wang $\mathrm{C}, \mathrm{Hu} \mathrm{C}$, Zhang R, Bao Y, Ma X, Lu J et al. Common variants of hepatocyte nuclear factor 1 beta are associated with type 2 diabetes in a Chinese population. Diabetes 2009; 58 : 1023-1027.

13. Bonnycastle LL, Willer CJ, Conneely KN, Jackson AU, Burrill CP, Watanabe RM et al. Common variants in maturity-onset diabetes of the young genes contribute to risk of type 2 diabetes in Finns. Diabetes 2006; 55: 2534-2540.

14. Stancakova A, Paananen J, Soininen P, Kangas AJ, Bonnycastle LL, Morken MA et al. Effects of 34 risk loci for type 2 diabetes or hyperglycemia on lipoprotein subclasses and their composition in 6,580 nondiabetic Finnish men. Diabetes 2011; 60: 1608-1616.

15. Kopelman PG. Obesity as a medical problem. Nature 2000; 404: 635-643.

16. Haslam DW, James WP. Obesity. Lancet 2005; 366: 1197-1209.

17. Scherer PE. Adipose tissue: from lipid storage compartment to endocrine organ. Diabetes 2006; 55: 1537-1545.

18. Ouchi N, Parker JL, Lugus JJ, Walsh K. Adipokines in inflammation and metabolic disease. Nat Rev Immunol 2011; 11: 85-97.

19. Gimeno RE, Klaman LD. Adipose tissue as an active endocrine organ: recent advances. Curr Opin Pharmacol 2005; 5: 122-128.

20. Wang JT, Jiao P, Zhou Y, Liu Q. Protective effect of dihydromyricetin against lipopolysaccharide-induced acute kidney injury in a rat model. Med Sci Monit 2016; 22: 454-459.

21. Verhave JC, Bech AP, Wetzels JF, Nijenhuis T. Hepatocyte nuclear factor 1beta-associated kidney disease: more than renal cysts and diabetes. J Am Soc Nephrol 2016; 27: 345-353.

22. Kim EK, Lee JS, Cheong HI, Chung SS, Kwak SH, Park KS. Identification and functional characterization of P159L mutation in HNF1B in a family with Maturity-Onset Diabetes of the Young 5 (MODY5). Genomics Inform 2014; 12: 240-246.

23. Tjora $E$, Wathle G, Erchinger $F$, Engjom $T$, Molven $A$, Aksnes $L$ et al. Exocrine pancreatic function in hepatocyte nuclear factor 1beta-maturity-onset diabetes of the young (HNF1BMODY) is only moderately reduced: compensatory hypersecretion from a hypoplastic pancreas. Diabet Med 2013; 30: 946-955.

24. Kornfeld JW, Baitzel C, Konner AC, Nicholls HT, Vogt MC, Herrmanns K et al. Obesityinduced overexpression of miR-802 impairs glucose metabolism through silencing of $\mathrm{Hnf} 1 \mathrm{~b}$. Nature 2013; 494: 111-115.

25. Powell K. Obesity: the two faces of fat. Nature 2007; 447: 525-527.

26. Houtkooper RH, Auwerx J. Obesity: new life for antidiabetic drugs. Nature 2010; 466: 443-444.

27. Scully T. Obesity. Nature 2014; 508: S49.

28. Heneka MT, Nicotera P. Thoughts on obesity and brain glucose. Cell 2016; 165: 773-775.

29. Poulos SP, Dodson MV, Culver MF, Hausman GJ. The increasingly complex regulation of adipocyte differentiation. Exp Biol Medicine (Maywood) 2016; 241: 449-456.

30. Wang X, Hai C. Redox modulation of adipocyte differentiation: hypothesis of "Redox Chain" and novel insights into intervention of adipogenesis and obesity. Free Rad Biol Med 2015; 89: 99-125.

31. Rosen ED, Spiegelman BM. Molecular regulation of adipogenesis. Annu Rev Cell Dev Biol 2000; 16: 145-171.

32. Lefterova MI, Lazar MA. New developments in adipogenesis. Trends Endocrinol Metab 2009; 20: $107-114$.

33. Rosen ED, MacDougald OA. Adipocyte differentiation from the inside out. Nat Rev Mol Cell Biol 2006; 7: 885-896. 
34. Rosen ED, Walkey CJ, Puigserver $\mathrm{P}$, Spiegelman BM. Transcriptional regulation of adipogenesis. Genes Dev 2000; 14: 1293-1307.

35. Lehmann JM, Moore LB, Smith-Oliver TA, Wilkison WO, Willson TM, Kliewer SA. An antidiabetic thiazolidinedione is a high affinity ligand for peroxisome proliferator-activated receptor gamma (PPAR gamma). J Biol Chem 1995; 270: 12953-12956.

36. Barak Y, Nelson MC, Ong ES, Jones YZ, Ruiz-Lozano P, Chien KR et al. PPAR gamma is required for placental, cardiac, and adipose tissue development. Mol Cell 1999; 4: 585-595.

37. Tontonoz P, Hu E, Devine J, Beale EG, Spiegelman BM. PPAR gamma 2 regulates adipose expression of the phosphoenolpyruvate carboxykinase gene. Mol Cell Biol 1995; 15: 351-357.

38. Villanueva CJ, Waki H, Godio C, Nielsen R, Chou WL, Vargas L et al. TLE3 is a dual-function transcriptional coregulator of adipogenesis. Cell Metab 2011; 13: 413-427.

39. Tang QQ, Otto TC, Lane MD. Mitotic clonal expansion: a synchronous process required for adipogenesis. Proc Natl Acad Sci USA 2003; 100: 44-49.

40. Tang QQ, Jiang MS, Lane MD. Repressive effect of Sp1 on the C/EBPalpha gene promoter: role in adipocyte differentiation. Mol Cell Biol 1999; 19: 4855-4865.

41. Tormos KV, Anso E, Hamanaka RB, Eisenbart J, Joseph J, Kalyanaraman B et al. Mitochondrial complex III ROS regulate adipocyte differentiation. Cell Metab 2011; 14: 537-544.
42. Lee $\mathrm{H}$, Lee YJ, Choi H, Ko EH, Kim JW. Reactive oxygen species facilitate adipocyte differentiation by accelerating mitotic clonal expansion. J Biol Chem 2009; 284: 10601-10609.

43. Wang X, Liu J, Long Z, Sun Q, Liu Y, Wang $L$ et al. Effect of diosgenin on metabolic dysfunction: role of ERbeta in the regulation of PPARgamma. Toxicol Appl Pharmacol 2015; 289: 286-296.

44. Wang $X$, Zhang W, Chen $\mathrm{H}$, Liao $\mathrm{N}$, Wang Z, Zhang $X$ et al. High selenium impairs hepatic insulin sensitivity through opposite regulation of ROS. Toxicol Lett 2014; 224: 16-23.

45. Wang X, Yang P, Liu J, Wu H, Yu W, Zhang T et al. RARgamma-C-Fos-PPARgamma2 signaling rather than ROS generation is critical for all-trans retinoic acid-inhibited adipocyte differentiation. Biochimie 2014; 106: 121-130.

46. Tang QQ, Zhang JW, Daniel Lane M. Sequential gene promoter interactions of C/EBPbeta, C/EBPalpha, and PPARgamma during adipogenesis. Biochem Biophys Res Commun 2004; 319: 235-239.

47. Wang X, Liu R, Zhang W, Zhang X, Liao N, Wang Z et al. Oleanolic acid improves hepatic insulin resistance via antioxidant, hypolipidemic and anti-inflammatory effects. Mol Cell Endocrinol 2013; 376: 70-80

Supplementary Information accompanies this paper on Cell Death and Differentiation website (http://www.nature.com/cdd) 